\title{
Cytogenetics and Genome Size Evolution in Illicium L.
}

\author{
Thomas G. Ranney ${ }^{1,5}$, Connor F. Ryan ${ }^{2}$, Lauren E. Deans ${ }^{3}$, \\ and Nathan P. Lynch ${ }^{4}$ \\ Mountain Crop Improvement Lab, Department of Horticultural Science, \\ Mountain Horticultural Crops Research and Extension Center, \\ North Carolina State University, 455 Research Drive, Mills River, \\ NC 28759-3423
}

Additional index words. ANA grade basal angiosperms, chromosome numbers, DNA content, flow cytometry, plant breeding, reciprocal translocation, star anise, star flower shrub

\begin{abstract}
Illicium is an ancient genus and member of the earliest diverging angiosperms known as the Amborellales, Nymphaeales, and Austrobaileyales (ANA) grade. These adaptable, broadleaf evergreen shrubs, including $\approx 40$ species distributed throughout Asia and North America, are valued for diverse culinary, medicinal, and ornamental applications. The study of cytogenetics of Illicium can clarify various discrepancies and further elucidate chromosome numbers, ploidy, and chromosome and genome size evolution in this basal angiosperm lineage and provide basic information to guide plant breeding and improvement programs. The objectives of this study were to use flow cytometry and traditional cytology to determine chromosome numbers, ploidy levels, and relative genome sizes of cultivated Illicium. Of the 29 taxa sampled, including $\approx 11$ species and one hybrid, 2C DNA contents ranged from $24.5 \mathrm{pg}$ for Illicium lanceolatum to $27.9 \mathrm{pg}$ for Illicium aff. majus. The genome sizes of Illicium species are considerably higher than other ANA grade lineages indicating that Illicium went through considerable genome expansion compared with sister lineages. The New World sect. Cymbostemon had a slightly lower mean $2 \mathrm{C}$ genome size of $25.1 \mathrm{pg}$ compared with the Old World sect. Illicium at $25.9 \mathrm{pg}$, providing further support for recognizing these taxonomic sections. All taxa appeared to be diploid and $2 n=2 x=28$, except for Illicium floridanum and Illicium mexicanum which were found to be $2 n=2 x=26$, most likely resulting from dysploid reduction after divergence into North America. The base chromosome number of $x=14$ for most Illicium species suggests that Illicium are ancient paleotetraploids that underwent a whole genome duplication derived from an ancestral base of $x=7$. Information on cytogenetics, coupled with phylogenetic analyses, identifies some limitations, but also considerable potential for the development of plant breeding and improvement programs with this genus.
\end{abstract}

Illicium, previously considered as the sole genus in Illiiaceae, has more recently been placed in the Schisandraceae within the order Austrobaileyales (The Angiosperm Phylogeny Group, 2016). The Austrobaileyales, along with the orders Nymphaeales and

Received for publication 25 Jan. 2018. Accepted for publication 25 Jan. 2018.

This work was funded, in part, by the North Carolina Agricultural Research Service (NCARS), Raleigh, NC, the North Carolina Biotechnology Center, Research Triangle Park, NC, the North Carolina Nursery and Landscape Association, Raleigh, NC, and the Kenan Institute, Raleigh, NC. Plant material was graciously provided by the JC Raulston Arboretum, Raleigh, NC; Dan Hinkley, Indianola, WA; Rick Crowder, Hickory, NC; and Ron Miller, Pensacola, FL.

We express our sincere thanks to Tom Eaker, Joel Mowrey, Andra Nus, and the staff at the Mountain Horticultural Crops Research and Extension Center for their technical assistance.

${ }^{1} \mathrm{JC}$ Raulston Distinguished Professor.

${ }^{2}$ Research Intern.

${ }^{3}$ Graduate Research Assistant.

${ }^{4}$ Research Specialist.

${ }^{5}$ Corresponding author. E-mail: tom_ranney@ncsu. edu.
Amborellales, form the most basal branches of the angiosperm phylogeny, cumulatively referred to as the ANA grade (VialetteGuiraud et al., 2011), and have origins dating back to the Late Jurassic to Early Cretaceous $\approx 160-130$ million years ago (Soltis et al., 2008). The ancient origin of ANA grade angiosperms (including Illicium), morphological similarity with early fossils, and limited molecular divergence suggests that these lineages may provide features and insights into the foundational traits of early angiosperms (Morris et al., 2007; Soltis et al., 2009).

Chromosome numbers and nuclear genome sizes vary widely among angiosperms. The original base chromosome number of the angiosperm linage was most likely somewhere between $x=6$ and 9 (Ehrendorfer et al., 1968; Raven, 1975; Stebbins, 1971) and increased over time with repeated cycles of whole genome duplication events (Soltis et al., 2003). Genomes can further expand through amplification of noncoding, repetitive DNA including retrotransposons (Leitch and Leitch, 2013). However, this "one-way ticket to genomic obesity" (Bennetzen and Kellogg, 1997) is often tempered by genome downsizing that can occur through recombination-based processes, such as unequal recombination and illegitimate recombination (Grover and Wendel, 2010; Soltis et al., 2015). There have only been limited reports on chromosome numbers and relative genome sizes for species and cultivars of Illicium. A base chromosome number of $x=14$ and diploidy has been reported for Illicium anisatum, Illicium parviflorum, Illicium ternstroemioides, and Illicium verum (Baolian, 1990; Lepper, 1982; Lin, 1989; Stone and Freeman, 1968; Whitaker, 1933). However, conflicting chromosome counts for I. floridanum exist, with different sources reporting a base chromosome number of either $x=13$ (Stone, 1965; Stone and Freeman, 1968) or $x=14$ (Whitaker, 1933). Reports of genome sizes for Illicium are also limited and variable. Nagl et al. (1977) reported a 2C genome size of $6.72 \mathrm{pg}$ (determined with scanning densitometry of Feulgen-stained nuclei) whereas Pellicer et al. (2013, Supplemental Table 2) reported genome sizes for Illicium henryi and Illicium simonsii to be 29.3 and $29.2 \mathrm{pg}$, respectively (determined with flow cytometry). Additional study of cytogenetics of Illicium can clarify various discrepancies and further elucidate chromosome and genome size evolution in this basal angiosperm lineage.

After many millions of years of divergence, there are now $\approx 40$ extant species of shrubs and small trees within the genus Illicium (Morris et al., 2007), including six found in the New World and the remaining species distributed throughout Asia (Shu, 2008; Vincent, 1997). Parsing the taxonomy and systematics of Illicium has been challenging because of the somewhat surprising morphological similarities between species despite the age and broad distribution of the genus (Morris et al., 2007). Molecular phylogeny studies have helped to clarify some species relationships (Hao et al., 2000; Morris et al., 2007; Oh et al., 2003). Morris et al. (2007), in agreement with Hao et al. (2000), provided a revised sectional classification of the genus with two sections: sect. Illicium (including the Old World species) and sect. Cymbostemon (including the New World species). Within sect. Cymbostemon there was also strong support for separation between the I. floridanum $+I$. mexicanum clade and the I. parviflorum + Illicium hottense + Illicium cubense + Illicium ekmanii clade.

Illicium are also of interest because of their unique and diverse plant metabolites that have both medicinal and culinary uses. Certain species of Illicium have been used in traditional medicine for treating pain, rheumatism, and skin inflammation (Liu et al., 2009). Most plant organs of Illicium are noticeably pungent with a strong odor of anise/terpenes. Extensive studies have been conducted to identify these compounds that include prenylated $\mathrm{C}_{6}-\mathrm{C}_{3}$ compounds, neolignans, and secoprezizaane-type sesquiterpenes that are found exclusively in Illicium (Liu et al., 2009). Many of these compounds and/or crude extracts from Illicium are 
biologically active and demonstrate antibacterial, anticancer, anti-inflammatory, antioxidant, antiviral (antiHIV), insecticidal, neurotoxic, neurotrophic, and phytotoxic activities (Liu et al., 2009). Illicium verum is of importance as a crop and the seedpods are well known in kitchens as the spice star anise (not to be confused with other species, which have a similar appearance and can be toxic). However, most I. verum grown as a crop is used as a source of shikimic acid and is the primary ingredient in the antiflu medication oseltamivir phosphate, sold as Tamiflu ${ }^{\circledR}$ (Wang et al., 2011).

Illicium have a suite of desirable ornamental qualities that make them valuable as nursery and landscape crops. Many species have attractive, tropical-looking evergreen leaves and distinctive showy flowers in whites, pinks, and reds in spring and sum$\mathrm{mer} /$ fall. They are also broadly adaptable with some taxa being cold hardy to USDA zone 6, tolerant of shady and wet sites (Griffin et al., 2004), and resistant to many diseases and pests, most notably deer. Nonetheless, Illicium are relatively uncommon in American landscapes and few cultivars exist for taxa beyond the three North American native species, I. floridanum, I. mexicanum, and I. parviflorum, and the Asian I. anisatum. Fewer interspecific hybrids exist and only $I$. floridanum $\times I$. mexicanum selections are currently available in the horticultural trade. Additional data on cytogenetics, including chromosome numbers and ploidy, of taxa in this genus would provide basic information to better enable plant breeding and improvement programs.

The objectives of this study were to determine chromosome numbers, ploidy levels, and relative genome sizes of cultivated Illicium taxa to gain further insights into the evolution, systematics, and the potential for interspecific hybridization.

\section{Materials and Methods}

Cytology. Chromosome counts were determined for six species representing major phylogenetic clades and sub-clades (Morris et al., 2007; Oh et al., 2003) to verify chromosome numbers and to calibrate genomes size measurements with ploidy levels. Actively growing root tips were excised and placed in a mixture of $4 \mathrm{~mm} 8$-hydroxyquinoline and $0.249 \mathrm{~mm}$ cycloheximide for $3 \mathrm{~h}$ at room temperature and then three to five additional hours in the dark at $4{ }^{\circ} \mathrm{C}$. Roots were placed into $3 \mathrm{~mL}$ of Carnoy's solution (six 95\% ethanol: three chloroform: one glacial acetic acid by volume) until the following morning, when the roots were stored in $70 \%$ ethanol. Fixed roots were hydrolyzed in a $3: 195 \%$ ethanol: $12 \mathrm{M} \mathrm{HCl}$ solution for $\approx 6-15 \mathrm{~min}$. Illicium anisatum Crowder \#2 (2007-117) and I. floridanum 'Swamp Hobbit' (2009-148) were then stained with a modified carbol fuchsin stain for at least 5 min (Kao, 1975). Because of poor staining with carbol fuchsin, I. henryi (1996-039), I. parviflorum 'Forest Green' (1998-329), I. mexicanum 'Aztec Fire'
(1996-041), and I. verum (2007-063) were stained with $1 \%$ acetocarmine stain for at least $5 \mathrm{~min}$ after which the root tips were sectioned onto a slide with a drop of stain, overlaid with a cover slip, and heated warm to the touch (Singh, 2003). Root tips were squashed underneath a cover slip on a microscope slide, and chromosomes were counted under oil immersion at $\times 1000$ magnification.

Flow cytometry. Relative 2C genome sizes were determined using a flow cytometer (Partec PA-II; Partec, Münster, Germany). Leaf tissue from 29 Illicium taxa was obtained from the JC Raulston Arboretum, Raleigh, NC; Mountain Crop Improvement Laboratory, Mills River, NC; Dan Hinkley, Indianola, WA; and Ken Cox and Steve Hootman, Federal Way, WA (Table 1). Young leaf tissue of Illicium samples and an internal standard [Pisum sativum 'Ctirad' 2C DNA $=8.76 \mathrm{pg}$ (Greilhuber et al., 2007)] were finely chopped together using a razor blade in a petri dish containing $400 \mu \mathrm{L}$ of nuclei extraction buffer (CyStain ultraviolet Precise P Nuclei Extraction Buffer; Sysmex Partec, Görlitz, Germany). The chopped sample and internal standard were then filtered through a $50-\mu \mathrm{m}$ nylon mesh filter into a test tube and stained with $1600 \mu \mathrm{L}$ of $4^{\prime}$, 6-diamidino-2-phenylindole (DAPI) staining buffer (Cystain ultraviolet Precise P Staining Buffer; Sysmex Partec) immediately before analysis. The flow cytometer was used to process the stained nuclei, with at least 3000 counts per subsample, two subsamples per taxon, and a $\mathrm{CV}$ less than $5 \%$ where possible.

Table 1. Relative 2C genome sizes for Illicium taxa.

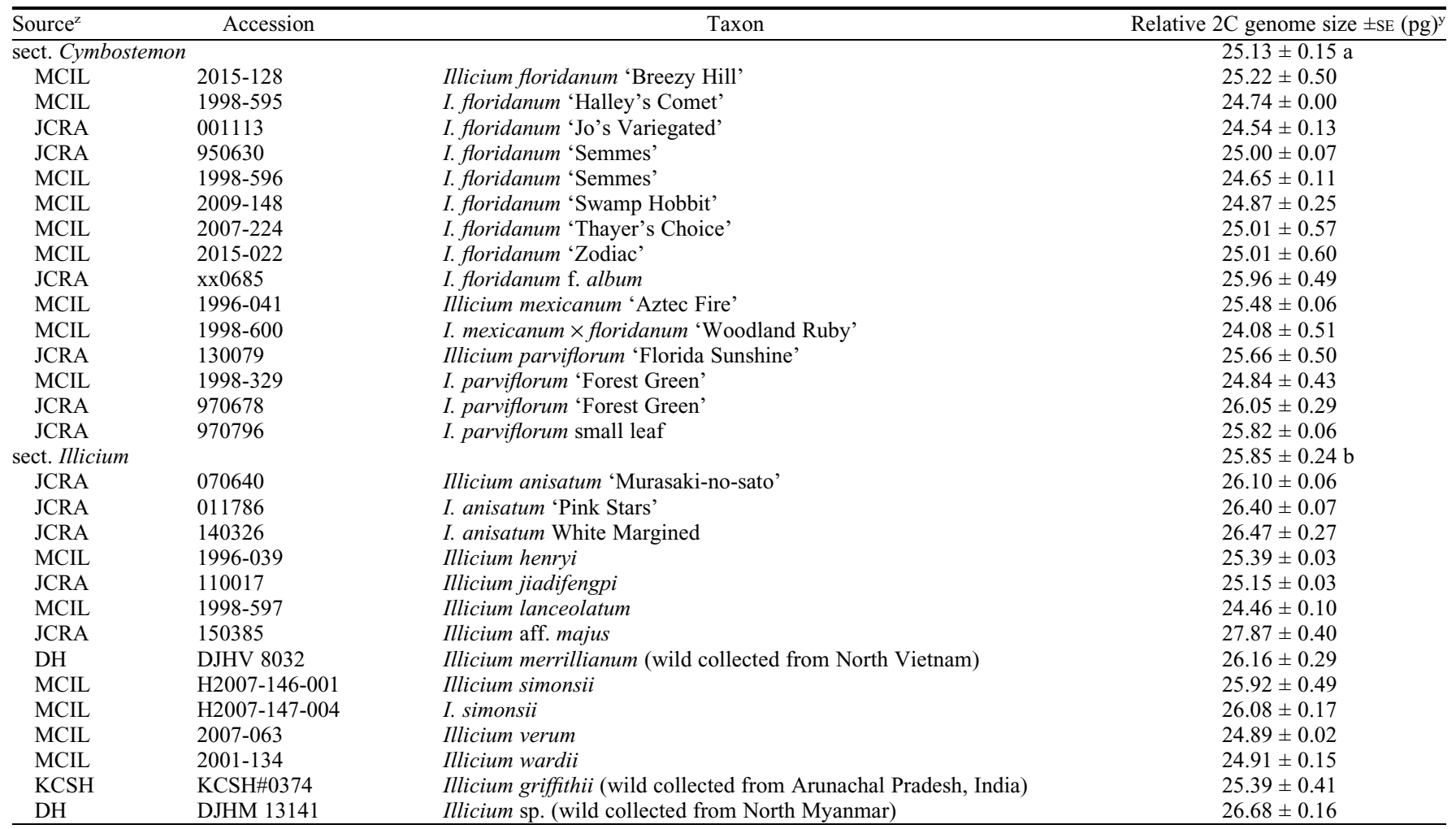

${ }^{\mathrm{z}} \mathrm{DH}=$ Dan Hinkley, Indianola, WA; JCRA = JC Raulston Arboretum, Raleigh, NC; KCSH = Ken Cox and Steve Hootman, Federal Way, WA; and MCIL = Mountain Crop Improvement Laboratory, Mills River, NC.

${ }^{\mathrm{y}}$ Overall means for sect. Cymbostemon (25.13 pg) (a) and sect. Illicium $(25.85 \mathrm{pg}$ ) (b) were significantly different at $P \leq 0.01$. 
Genome size $(2 \mathrm{C})$ of samples was calculated as: $2 \mathrm{C}=$ genome size of standard $\times$ (mean fluorescence value of sample $\div$ mean florescence value of standard). The experimental design was completely randomized.

Data for $2 \mathrm{C}$ genome sizes were subjected to analysis of variance as a function of taxonomic sections (sect. Cymbostemon and sect. Illicium) and representative species of clades within sect. Cymbostemon [(I. floridanum $+I$. mexicanum) and (I. parviflorum)] (Proc GLM; SAS Version 9.2; SAS Inst., Cary, NC).

\section{Results and Discussion}

Chromosome counts of accessions of llicium sect. Illicium taxa, including I. anisatum, I. verum, and I. henryi (Fig. 1A-C), were $2 n=2 x=28$. Although, chromosome counts have not been reported previously for $I$. henryi, these results are consistent with prior reports for I. anisatum and I. verum (Baolian, 1990; Whitaker, 1933). Chromosome counts for Illicum sect. Cymbostemon were more variable. Illicium parviflorum (Fig. 1D), was found to be $2 n=2 x=28$, substantiating
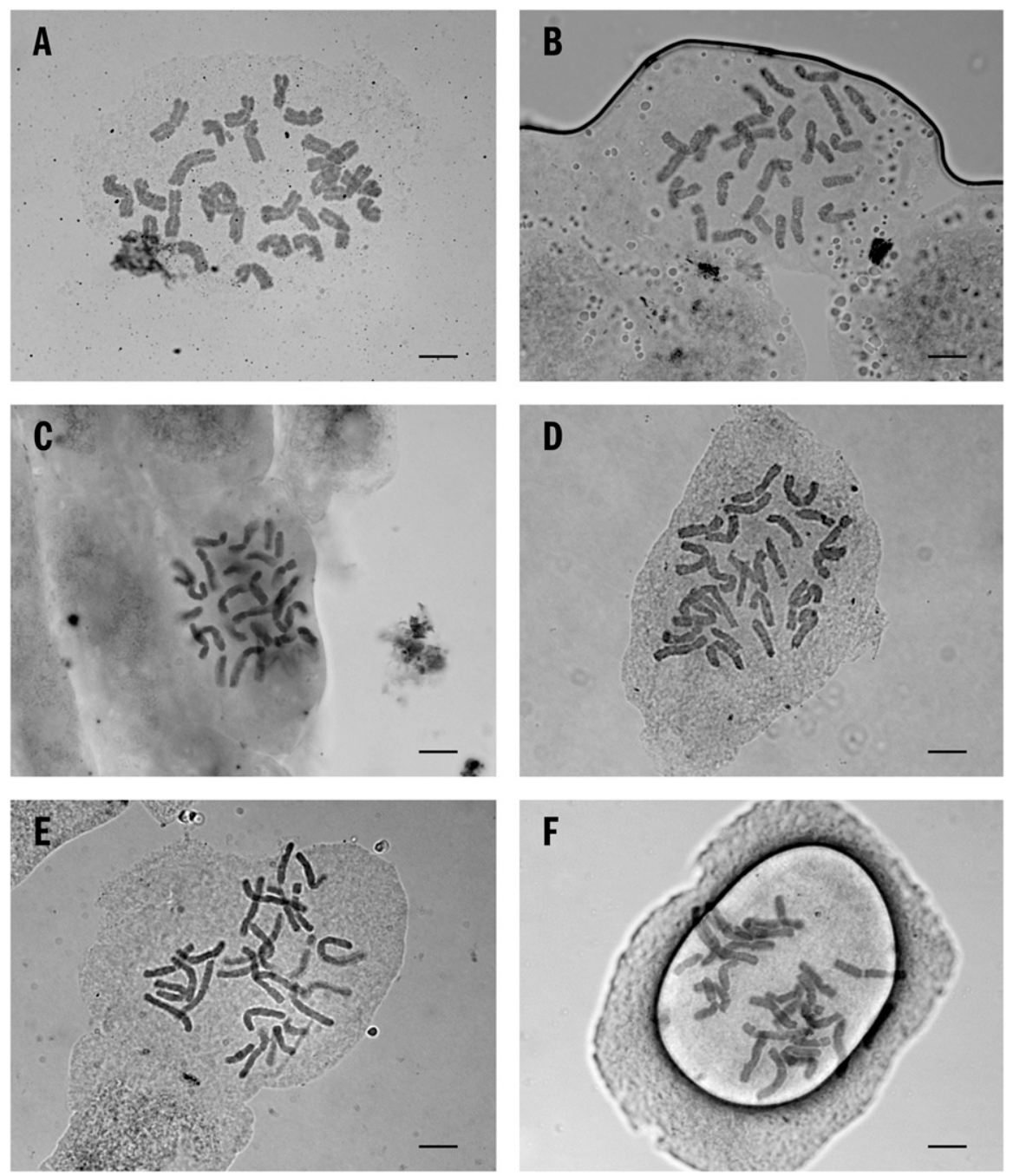

Fig. 1. Chromosomes of (A) Illicium anisatum $(2 n=28)$, (B) Illicium verum $(2 n=28)$, (C) Illicium henryi $(2 n=28)$, (D) Illicium parviflorum $(2 n=28),(\mathbf{E})$ Illicium floridanum $(2 n=26)$, and $(\mathbf{F})$ Illicium mexicanum $(2 n=26)$. Bar $=15.86 \mu \mathrm{m}$. event occurred before diversification of the New World crown group, estimated by Morris et al. (2007) to be a minimum age of 5 million years. The reduced base karyotypes of I. floridanum and I. mexicanum probably resulted more recently from dysploid reduction resulting from a reciprocal translocation (chromosomal fusion), yielding $x=13$ (Ehrendorfer et al., 1968; Schubert and Lysak, 2011).

Implications of this research for breeding and crop improvement suggest there is wide similarity in chromosome numbers and ploidy within the genus, except for I. floridanum and I. mexicanum. The close relationship between these two species and unique chromosome numbers helps explain their ability to produce fertile hybrids (T.G. Ranney, personal observation), but most likely will limit their potential to produce viable hybrids with species with $2 n=2 x=28$. Selected crosses between species within sect. Illicium have been successful, including I. anisatum $\times$ wardii and I. anisatum $\times$ simonsii (T.G. Ranney, personal observation), which is not unexpected knowing that they share similar chromosome numbers and ploidy and are placed in the same phylogenetic clade/ section. These results and observations provide hope that many of the sect. Illicium species may hybridize, allowing for breeding programs to improve these crops for medicinal, culinary, and ornamental applications. The potential for breeding between sections has yet to be determined.

Relative 2C genome sizes of 29 taxa representing $\approx 11$ species and one hybrid of Illicium were surprisingly similar and ranged from $24.5 \mathrm{pg}$ in I. lanceolatum to $27.9 \mathrm{pg}$ in I. aff. majus (Table 1) and indicates a common ploidy for all Illicium species. The substantially higher genome size of $I$. aff. majus is of interest and may warrant a separate chromosome count to verify the base chromosome number in that taxon. Overall, these values were similar, but slightly lower than those values reported (Pellicer et al., 2013, Supplemental Table 2) for I. henryi and I. simonsii reported as 29.3 and $29.2 \mathrm{pg}$, respectively [determined with propidium iodide (PI stain)]. Different fluorochrome stains may give slightly different estimates of genome size, though both PI and DAPI have been found to be effective and consistent for determining and comparing ploidy levels and relative genome size among closely related taxa (Parris et al., 2010). Furthermore, DAPI typically provides more precise and repeatable results as it is specific to double-stranded DNA and is not influenced by variable chromatin structure whereas fluorescence of PI is susceptible to staining inhibitors and their antagonists (Doležel and Bartoš, 2005; Greilhuber et al., 2007). We determined the relative genome size of I. anisatum to be $26.2 \mathrm{pg}$, nearly four times the value of $6.72 \mathrm{pg}$ reported by Nagl et al. (1977), who used scanning densitometry with Feulgen-stained nuclei. This discrepancy is most likely reflective of the more accurate and reliable measures provided by flow cytometry. 
Analysis of variance and comparison between sections showed that the New World sect. Cymbostemon had a slightly lower 2C genome size of $25.1 \mathrm{pg}$ compared with the Old World sect Illicium at $25.9 \mathrm{pg}$, providing additional credence to these sectional designations. Despite substantial karyotypic differences and varying base chromosome numbers, there was not a significant difference in $2 \mathrm{C}$ genome sizes between clades (I. floridanum + I. mexicanum) and (I. parviflorum) within sect. Cymbostemon. The genome sizes of Illicium species are considerably higher than other ANA grade lineages. Although Amborella, the sole genus in Amborellales has a $2 \mathrm{C}$ genome size of $1.8 \mathrm{pg}$, the Nymphaeales range from 0.9 to $9.3 \mathrm{pg}$, and the Austrobaileyales range from $8.2 \mathrm{pg}$ for Trimenia to as high as $29.3 \mathrm{pg}$ for Illicium (Pellicer et al., 2013). Compared with other ANA grade linages, Illicium went through a process of considerable genome expansion, including at least one whole genome duplication, and additional withinploidy genome increases. Despite this relatively large genome expansion, compared with the other ANA grade linages, it is somewhat surprising that genome sizes and ploidy levels of modern Illicium species are relatively conserved and appear somewhat static. Of course, many other angiosperm lineages have far exceeded the genome sizes of Illicium (e.g., Paris japonica with $2 \mathrm{C}=$ 304.5 pg, Pellicer et al., 2010).

The results of this study help to clarify and expand information on cytogenetics of Illicium. This information provides further insights into the evolution of chromosome numbers and genome sizes in this primitive, basal angiosperm lineage substantiating an ancestral base chromosome number of $x=7$, ancient whole genome duplication, dysploid reduction in multiple species, substantial genome expansion compared with sister lineages, and classification of two taxonomic sections within the genus. Specific information on ploidy and chromosomes numbers, coupled with phylogenetic analyses, identifies some limitations, but also considerable potential for the development of breeding and improvement programs with this genus.

\section{Literature Cited}

Baolian, S. 1990. Karyotype analysis of Illicium verum Hook. f. J. Southwest For. College 10(1):56-59.
Bennetzen, J.L. and E.A. Kellogg. 1997. Do plants have a one-way ticket to genomic obesity? Plant Cell 9:1509-1514.

Doležel, J. and J. Bartoš. 2005. Plant DNA flow cytometry and estimation of nuclear genome size. Ann. Bot. 95:99-100.

Ehrendorfer, F., F. Krendl, E. Habeler, and W. Sauer. 1968. Chromosome numbers and evolution in primitive angiosperms. Taxon 17(4):337-353.

Greilhuber, J., E.M. Temsch, and J.C.M. Loureiro, 2007. Nuclear DNA content measurement, p. 67-101. In: J. Doležel, J. Greilhuber, and J. Suda (eds.). Flow cytometry with plant cells: Analysis of genes, chromosomes and genomes. Wiley-VCH, Weinheim, Germany.

Griffin, J.J., T.G. Ranney, and D.M. Pharr. 2004. Photosynthesis, chlorophyll fluorescence, and carbohydrate content of Illicium taxa grown under varied irradiance. J. Amer. Soc. Hort. Sci. 129:46.

Grover, C. and J.F. Wendel. 2010. Recent insights into mechanisms of genome size change in plants. J. Bot. 2010:1-8.

Hao, G., R.M.K. Saunders, and M. Chye. 2000. A phylogenetic analysis of the Illiciaceae based on sequences of internal transcribed spacers (ITS) of nuclear ribosomal DNA. Plant Syst. Evol. 223(1-2):81-90.

Kao, K.N. 1975. A nuclear staining method for plant protoplasts, p. 60-62. In: O.L. Gamborg and L.R. Wetter (eds.). Plant tissue culture methods. Natl. Res. Council Canada, Prairie Reg. Lab., Saskatoon, Canada.

Leitch, I.J. and A.R. Leitch. 2013. Genome size diversity and evolution in land plants, p. 307322. In: I.J. Leitch, J. Greilhuber, J. Dolezel, and J. Wendel (eds.). Plant genome diversity, Vol. 2. Springer-Verlag, Wien, Germany.

Lepper, L. 1982. Beiträge zur chromosomedokumentation cubanischer pflanzensippen. Revista Jard. Bot. Nac. Univ. Habana 3:71-102.

Lin, Q. 1989. A study on the karyotype of Illicium ternstroemioides. Acta Bot. Austro Sin. 4:85-89.

Liu, Y-N., X-H. Su, C-H. Hou, X-P. Zhang, Q-W. Shi, and Y-C. Gu. 2009. Chemical constituents of plants from the genus Illicium. Chem. Biodivers. 6:963-989.

Morris, A.B., C.D. Bell, J.W. Clayton, W.S. Judd, D.E. Soltis, and P.S. Soltis. 2007. Phylogeny and divergence time estimation in Illicium with implications for new world biogeography. Syst. Bot. 32(2):236-249.

Nagl, W., T. Habermann, and H. Fusenig. 1977. Nuclear DNA contents in four primitive angiosperms. Plant Syst. Evol. 127(2-3):103-105.

Oh, I-C., T. Denk, and E.M. Friis. 2003. Evolution of Illicium (Illiciaceae): Mapping morphological characters on the molecular tree. Plant Syst. Evol. 240:175-209.

Parris, J.K., T.G. Ranney, H.T. Knap, and W.V. Baird. 2010. Ploidy levels, relative genome sizes, and base pair composition in magnolia. J. Amer. Soc. Hort. Sci. 135(6):533-547.
Pellicer, J., M.F. Fay, and I.J. Leitch. 2010. The largest eukaryotic genome of them all? Bot. J. Linn. Soc. 164:10-15.

Pellicer, J., L.J. Kelly, C. Magdalena, and I.J. Leitch. 2013. Insights into the dynamics of genome size and chromosome evolution in the early diverging angiosperm lineage Nymphaeales (water lililies). Genome 56:1-13.

Raven, P.H. 1975. The basis of angiosperm phylogeny: Cytology. Ann. Missouri Bot. Garden 62:724-764.

Schubert, I. and M.A. Lysak. 2011. Interpretation of karyotype evolution should consider chromosome structural constraints. Trends Genet. 27(6):207-216.

Shu, B.J. 2008. Illiciacea. Flora China 7:32-38.

Singh, R.J. 2003. Plant cytogenetics. $2^{\text {nd }}$ ed. CRC Press LLC, Boca Raton, FL.

Soltis, D.E., C.D. Bell, S. Kim, and P.S. Soltis. 2008. Origin and early evolution of angiosperms. Ann. NY. Acad. Sci. 1133:3-25.

Soltis, D.E., P.S. Soltis, M.D. Bennett, and I.J. Leitch. 2003. Evolution of genome size in the angiosperms. Amer. J. Bot. 90(11):15961603.

Soltis, P.S., S.F. Brockington, M-J. Yoo, A. Piedrahita, M. Latvis, M.J. Moore, A.S. Chanderbali, and D.E. Soltis. 2009. Floral variation and floral genetics in basal angiosperms. Amer. J. Bot. 96(1):110-128.

Soltis, P.S., D.B. Marchant, Y. Van de Peer, and D.E. Soltis. 2015. Polyploidy and genome evolution in plants. Curr. Opin. Genet. Dev. 35:119-125.

Stebbins, G.L. 1971. Chromosomal evolution in higher plants. Addison-Wesley, London.

Stone, D.E. 1965. In documented chromosome numbers of plants. Madrono 18:122-126.

Stone, D.E. and J.L. Freeman. 1968. Cytotaxonomy of Illicium floridanum and I. parviflorum (Illiciaceae). J. Arnold Arbor. 49:41-51.

The Angiosperm Phylogeny Group. 2016. An update of the angiosperm phylogeny group classification for the orders and families of flowering plants: APG IV. Bot. J. Linn. Soc. 181:1-20.

Vialette-Guiraud, A.C.M., M. Alaux, F. Legeai, C. Finet, P. Chambrier, S.C. Brown, A. Chauvet, C. Magdalena, P.J. Rudall, and C.P. Scutt. 2011. Cabomba as a model for studies of early angiosperm evolution. Ann. Bot. 108:589-598.

Vincent, M.A. 1997. Illiciaceae (de Candolle) A. C. Smith. In: Flora of North America Editorial Committee (ed.). Flora of North America, Vol. 3, Oxford. 11 Jan. 2018. <http://www.efloras. org/florataxon.aspx?flora_id=1\&taxon_id=10450>.

Wang, G., W. Hu, B. Huang, and L. Qin. 2011. Illicium verum: A review on its botany, traditional use, chemistry and pharmacology. J. Ethnopharmacol. 136(1):10-20.

Whitaker, T.W. 1933. Chromosome number and relationship in the Magnoliales. J. Arnold Arbor. 14:376-385. 